\title{
ASPECTOS ÉTICOS DEL USO DE IDENTIFICADORES BIOMÉTRICOS
}

\author{
Boris A. Lucero M. ${ }^{1}$, Chiara Saracini ${ }^{1}$, Marco Mora ${ }^{2}$, María Teresa Muñoz-Quezada ${ }^{1}$
}

Resumen: La biometría ha tenido una rápida expansión como tecnología, siendo cada vez más accesible para el público general, prestando utilidad a los gobiernos y a la sociedad en el manejo de la seguridad ciudadana. Se aplica ya sea para la verificación o para la identificación de la identidad de un individuo y, desde un punto de vista puramente técnico, constituye un problema de reconocimiento de patrones. En Chile, el marco legal respecto del manejo de datos personales no incluye a la biometría en específico en su regulación. A la fecha, se encuentra en trámite un nuevo proyecto de ley que considera tanto los datos biométricos como los perfiles biológicos. En otros países, los informes de las últimas dos décadas, que analizan el uso de biometría, plantean como principal foco de preocupación, desde el punto de vista ético, el derecho a la privacidad de los individuos, entregándose orientaciones prácticas y discusiones en cuanto a elementos necesarios para la aplicación apropiada de estas tecnologías novedosas. Finalmente, se contextualiza desde un breve análisis de la biopolítica, el papel que cumple la biometría y los requerimientos éticos básicos para su desarrollo en dicho marco.

Palabras clave: biometría, bioseñales, datos personales, derecho a la privacidad

\section{Bioethical aspects of the use of biometric identifiers}

\begin{abstract}
Biometrics has had a rapid expansion as a technology, being increasingly accessible to the public, and lending utility to governments and society in the management of citizen security. It is applied either for verification or for identification of an individual's identity and technically constitutes a problem of pattern recognition. In Chile, the existing legal framework regarding the handling of personal data does not include specific biometrics in its regulation. A new bill that considers both biometric data and Biological profiles is in process. In addition, we revised the international reports of the last two decades that analyze the use of biometrics. The primary focus of concern of these documents, from the ethical point of view, is the right to privacy of individuals, giving practical guidance and discussion regarding the elements necessary for the application of these novel technologies in an appropriate ethically manner. Finally, we contextualize from a brief analysis of biopolitics the role of biometrics and the basic ethical requirements for its development in that framework.
\end{abstract}

Key words: biometrics, bio-signals, personal data, right to privacy

\section{Aspectos éticos do uso de identificadores biométricos}

Resumo: A biometria teve uma rápida expansão como tecnologia, sendo cada vez mais acessível para o público geral, sendo de utilidade para os governos e à sociedade no manejo da segurança cidadã. Ela pode ser aplicada para a verificaçáo ou estabelecimento da identidade de um indivíduo e, desde um ponto de vista puramente técnico, constitui um problema de reconhecimento de padróes. No Chile, o contexto legal acerca do manejo de dados pessoais náo inclui especificamente a biometria. Atualmente, encontra-se tramitando um novo projeto de lei que considera tanto os dados biométricos como os perfis biológicos. Em outros países, os informes das últimas duas décadas que analisam o uso da biometria, propóem como principal foco de preocupação, do ponto de vista ético, o direito à privacidade dos indivíduos, entregando-se orientaçóes práticas acerca da aplicação apropriada destas tecnologias. Finalmente, desde uma breve análise da bio-política, contextualiza-se o papel que cumpre a biometria e os requerimentos éticos básicos para seu desenvolvimento em tal contexto legal.

Palavras chave: biometria, bio-sinais, dados pessoais, direito à privacidade

\footnotetext{
${ }^{1}$ Centro de Investigación en Neuropsicología y Neurociencias Cognitivas, Facultad Ciencias de la Salud, Universidad Católica del Maule, Chile Correspondencia: balucero@gmail.com

${ }^{2}$ Laboratorio de Investigaciones Tecnológicas en Reconocimiento de Patrones, Facultad Ciencias de la Ingeniería, Universidad Católica del Maule, Chile
} 


\section{Introducción}

La biometría, como campo de estudio y de desarrollo tecnológico, ha sido concebida conceptualmente desde alrededor de cuarenta ańos. En dicha época, algunas empresas informáticas empezaron a investigar la posibilidad de identificar a personas por medio de máquinas(1). La biometría, como disciplina, ha sido definida como la ciencia para establecer la identidad de un individuo según atributos físicos, químicos, o comportamentales(2). La necesidad de desarrollar sistemas a gran escala para el manejo de la identidad de los individuos en las sociedades modernas, así como también por el desarrollo de sistemas de seguridad más sofisticados, ha potenciado el desarrollo de este campo de investigación, sobre todo desde el punto de vista aplicado.

Técnicamente, la biometría constituye un problema de reconocimiento de patrones, lo cual se aplica ya sea para la verificación o para la identificación de la identidad de un individuo. En el caso de la verificación ("`es esta persona quien dice ser?”), se realiza un reconocimiento de un patrón que se captura y se evalúa si tiene coincidencia con los datos biométricos del individuo almacenados en algún registro en una base de datos, siendo por tanto un método de pareamiento de datos 1:1. En tanto, la identificación (“¿quién es esta persona?”) consiste en la comparación de un patrón biométrico capturado de un individuo con todos los datos biométricos disponibles en una base de datos, siendo un método de pareamiento $1: \mathrm{n}$, siendo $\mathrm{n}$ el tamaño de la base de datos.

Como se puede desprender de lo anterior, la biometría implica el manejo de datos característicos, ya sea de un individuo particular o de un grupo de individuos, los cuales son almacenados por quien maneja dichos datos como una representación de un perfil biológico.

Usualmente la identificación de los individuos se puede realizar a través de(3): objetos (carnet de identidad, tarjeta de identificación, etc.); conocimientos (claves personales, preguntas secretas, etc.); atributos conductuales (firma, forma de teclear, etc.); atributos físicos (huellas dactilares, iris, retina, rostro, etc.); otros atributos (voz, $\mathrm{ADN}$, etc.).
Sin embargo, desde época reciente ha emergido un nuevo tipo de medidas biométricas: las bioseñales. Estas corresponden a señales fisiológicas que se producen a partir de los cambios electroquímicos en el organismo. Las bioseñales se difunden a través del cuerpo hasta llegar a la superficie de la piel, pudiéndose capturar a través de electrodos. Entre estas bioseñales encontramos el electrocardiograma (ECG), el electromiograma (EMG) y la actividad electro dérmica a partir de las glándulas sudoríparas (EDA). Las bioseñales han tenido un vertiginoso progreso debido a que se ha mejorado enormemente la confiabilidad de los sensores. Además, el desarrollo tecnológico ha posibilitado que sean más baratos y, por tanto, más accesibles, pudiendo además ahora funcionar sin necesidad de cables, siendo menos invasivos $(4,5)$. Una ventaja adicional de las bioseñales es que no pueden ser manipuladas, como sería el caso de los marcadores biométricos tradicionales.

De modo más específico, desde la década de los ochenta se han ido generando propuestas y métodos para el reconocimiento automático de personas basado en el uso de señales de electroencefalografía (EEG). Solo desde algunos años, no obstante, se ha dado un desarrollo importante desde el punto de vista tecnológico y metodológico(6).

En comparación a todos los distintos tipos de mediciones biométricas para reconocimiento de identidad, tales como la de reconocimiento facial, del iris, y el más tradicional de uso de las huellas dactilares, las señales de la onda cerebral registrados por medio de EEG poseen características que no comparten con medidas más comúnmente utilizadas. Esto ha llevado a un interés para su desarrollo como conocimiento aplicado en el área, entendiendo que constituyen marcadores biológicos que no están expuestos y, por tanto, no conllevan el riesgo de que puedan ser capturados por elementos externos, lo cual sí sería posible tanto en el rostro, el iris o las huellas dactilares, para su falsificación.

Además de lo anterior, el reconocimiento basado en la onda cerebral capturada con EEG no puede ser utilizado de manera coercitiva, siendo imposible que un tercero obligue a un usuario a autenticarse usando EEG. De exponerse a esto, se vería reflejado en el EEG con una señal que estaría al- 
terada, denegando el reconocimiento y no siendo útil de tal modo para su uso biométrico(7).

Existen diversas características que llevan a definir un identificador biométrico, entre las cuales están: universalidad, unicidad, permanencia, posibilidad de ser recolectado, desempeño, aceptabilidad y resistencia a ataques(8).

La universalidad se refiere a que todas las personas deben poseer la característica que se utilizará como medida biométrica; la unicidad, a su vez, presupone que, al comparar dos personas, el identificador biométrico debiera diferenciarlas a partir de cómo se manifiesta la característica considerada; la permanencia se refiere a que el criterio de coincidencia para la identificación corresponde a características suficientemente estables en el tiempo; la posibilidad de ser recolectado alude a que, en la práctica, la característica debe ser cuantitativamente medible con algún dispositivo; el desempeño implica que las tasas de reconocimiento con la medida son satisfactorias, para esto se utilizan medidas de desempeño, tales como la tasa de identificación genuina, la de identificación falsa, la de rechazo falso, entre otras; que sea resistente a ataques se entiende a partir de cuan robusto puede ser frente a tentativas por capturar el rasgo de manera furtiva por parte de terceros para su reproducción y su posterior uso fraudulento. Este elemento es el que representa el principal atractivo del uso de EEG en biometría, ya que corresponde a una actividad eléctrica cerebral espontánea de un órgano vivo en funcionamiento, lo cual no la hace reproducible como objeto, por su complejidad como señal.

La aceptabilidad, finalmente, se refiere a que el usuario no debiera tener una fuerte objeción respecto a las características de la medición del identificador en particular. En esta característica se podrían concentrar algunas de las consideraciones éticas más salientes para la proyección del uso del EEG como identificador biométrico. En primer término, porque pudiese evocar creencias usuales del sentido común respecto al EEG como una especie de "lector de la mente" y de análisis de las emociones o estados internos por parte de quien maneje los datos. Asimismo, podría ser objetado en cuanto al cuidado de la privacidad, ya que potencialmente podría llevar a detectar patologías o tendencias patológicas, pudiendo exponer a dis- criminación o perjuicio hacia la dignidad de las personas(9).

A partir de todo lo expuesto, y en consideración a la rápida difusión de la biometría y de las bioseñales, sobre todo en la últimas dos décadas, han empezado algunas discusiones (escasas aún) respecto de las implicancias sociales y bioéticas que conllevan estas tecnologías. El contexto histórico del desarrollo del campo de estudio de la biometría ha llevado a que, en pocas décadas, haya pasado desde un elemento narrativo en historias de ciencia ficción a una completa realidad en el campo aplicado, siendo cada vez más usada para la identificación de los usuarios y autenticación de sistemas informáticos, en controles fronterizos y para propósitos gubernamentales(10).

Sin embargo, en Chile esta es una temática que aún no ha sido abordada en específico. Sobre esta base, presentaremos algunas consideraciones que permiten poner en perspectiva y reflexionar respecto del marco ético y legal, tanto en Chile como en el extranjero, en la aplicación de la biometría y de las bioseñales para la identificación de los individuos que forman parte de la sociedad.

\section{Implicancias ético-legales de la biometría}

A partir de lo expuesto, se puede evidenciar que el auge de la biometría se asocia actualmente a la importancia que brindan las distintas naciones a la seguridad y al vertiginoso desarrollo de tecnologías, que la hacen una medición accesible y que puede facilitar el manejo de datos a gran escala. No obstante, este tipo de medidas biológicas podrían presentar una serie de desafíos en términos del respeto de los derechos individuales, específicamente en cuanto a la libertad de las personas, la protección de sus datos y la confidencialidad. Asimismo, podría resultar importante discutir cómo se da la relación entre derechos individuales y colectivos en la utilización de la biometría, ante la probable complejidad que significa satisfacer ambos tipos de derechos de manera equilibrada en su aplicación en la práctica.

\section{Marco regulatorio legal de Chile respecto a ma- nejo de datos personales}

En el marco legal chileno, la protección de los datos 
personales está regulada en la ley $\mathrm{N}^{\circ} 19.628(11)$. Esta considera como "datos de carácter personal los relativos a cualquier información concerniente a personas naturales, identificadas o identificables" (art. $2^{\circ}$, letra f). A su vez, el artículo $4^{\circ}$ especifica que la utilización de los datos personales es posible solo cuando la ley o el titular entregue expresamente su consentimiento para ello. Este mismo artículo señala que, aun cuando exista el consentimiento, se debe informar respecto del propósito del almacenamiento o eventual comunicación pública de los datos compartidos. Se detalla además que la autorización debe constar por escrito, la cual también puede ser revocada, pero sin efecto retroactivo. No obstante, esto no es completamente estricto, ya que se libera de la necesidad de esta autorización el uso para fines financieros y comerciales y por parte de personas jurídicas privadas para uso exclusivo suyo, con fines de tarifación, estadística u otro de beneficio general. Luego, el artículo $5^{\circ}$ también cautela de modo más preciso lo referido a requerimientos de datos personales mediante red electrónica, lo cual implicará la individualización del requirente, dejar constancia del motivo y propósito del requerimiento, y el tipo de datos que se transmitirán. Se establece que el receptor solo puede utilizar los datos personales para los fines que motivaron la transmisión; no obstante, aclarando que esto no aplicará en el caso que los datos personales sean accesibles al público general. Respecto de la eliminación de los datos, el artículo $6^{\circ}$ entrega referencias que llevan a entender que solo depende del criterio del responsable del banco de datos personales, sin establecer ningún plazo específico de caducidad. El artículo $7^{\circ}$ cautela que quienes trabajen en tratamiento de datos personales deben guardar secreto de los mismos, lo cual procede incluso una vez que han dejado de trabajar en dicha ocupación. No obstante, no se especifica ningún tipo de sanción frente al no cumplimiento de dicha condición de confidencialidad. Cabe consignar que la ley define el tratamiento de datos como "cualquier operación o complejo de operaciones o procedimientos técnicos, de carácter automatizado o no, que permitan recolectar, almacenar, grabar, organizar, elaborar, seleccionar, extraer, confrontar, interconectar, disociar, comunicar, ceder, transferir, transmitir, o cancelar datos de carácter personal, o utilizarlos en cualquier otra forma” (art. $2^{\circ}$, letra o).
Asociado de manera más directa a lo abordado aquí respecto de la biometría, es lo referido en la ley como "dato sensible". Esto corresponde legalmente a "aquellos datos personales que se refieren a las características físicas o morales de las personas, o a hechos o circunstancias de su vida privada o intimidad, tales como los hábitos personales, el origen racial, las ideologías y opiniones políticas, las creencias o convicciones religiosas, los estados de salud físicos o psíquicos y la vida sexual" (art. $2^{\circ}$, letra g). La ley contempla la regulación de este tipo de datos en el art. $10^{\circ}$, que establece que "no pueden ser objeto de tratamiento los datos sensibles, salvo cuando la ley lo autorice, exista consentimiento del titular o sean datos necesarios para la determinación u otorgamiento de beneficios de salud que corresponda a sus titulares".

Luego, la compleja relación entre los derechos individuales y colectivos en el tratamiento de los datos personales es abordada en el Título II de la ley. Si bien se contempla el privilegio de los derechos de los titulares ante los responsables de un banco de datos a solicitar, modificar, cancelar o bloquear la información de los datos personales sin limitación alguna (art. 13); no obstante, casi a continuación (art. 15) se establece una excepción al derecho individual recién mencionado, cuando impida o entorpezca el debido cumplimiento de funciones fiscalizadoras de organismos públicos, afecte el secreto o reserva de alguna disposición legal o reglamento, o la seguridad de la nación o el interés nacional.

En cuanto al manejo de datos de salud, también resulta de interés lo consignado en el Título Final de la ley, respecto a que "las recetas médicas y análisis o exámenes de laboratorios clínicos y servicios relacionados con la salud son reservados. Sólo podrá revelarse su contenido o darse copia de ellos con el consentimiento expreso del paciente, otorgado por escrito...". En el contexto de la temática aquí abordada, esto lleva a entender que no resultaría plausible, desde el punto de vista legal, el uso de bioseñales obtenidas en exámenes de salud para su uso en identificación o verificación de identidad por biometría.

Actualmente, y desde el año 2017, existe en estudio un nuevo proyecto de ley(12), aprobado ya por la Cámara de Diputados en 2018, que busca 
actualizar a los requerimientos internacionales y las nuevas tecnologías el marco legal que rige a la fecha, de modo de fortalecer la protección de los intereses y el derecho a la privacidad de los individuos frente al uso extendido y cotidiano de plataformas y formularios digitales. No obstante, un antecedente importante para esta temática es que el artículo $3^{\circ}$ de este proyecto considera explícitamente, dentro de la definición de lo que es un dato sensible, los datos biométricos. Estos, en el nuevo proyecto de ley, son definidos como "datos personales obtenidos a partir de un tratamiento técnico específico, relativos a las características físicas, fisiológicas o conductuales de una persona natural, que permitan o confirmen la identificación única de dicha persona, como imágenes faciales o datos dactiloscópicos". Asimismo, el proyecto precontempla un inciso específico íntegramente dedicado a regular los datos personales biométricos (artículo 16ter), el cual estipula:

"El responsable que trate datos personales biométricos, tales como la huella digital, el iris, los rasgos de la mano o faciales y la voz, deberá proporcionar al titular la siguiente información específica: a) la identificación del sistema biométrico usado; b) la finalidad específica para la cual los datos recolectados por el sistema biométrico serán utilizados; c) el período durante el cual los datos biométricos serán utilizados; d) la forma en que el titular puede ejercer sus derechos.

Un reglamento regulará la forma y los procedimientos que se deben utilizar para la implementación de los sistemas biométricos.

Con todo, no se podrán crear o mantener bancos de huellas digitales o de otros datos biométricos, salvo expresa autorización legal”.

Lo anterior evidencia que el proyecto exige la aplicación de procedimientos biométricos con el respeto del derecho a ser informado, y el conocimiento respecto de un reglamento que se presupone como objeto orientador y guía en la implementación de las técnicas biométricas.

Por otra parte, resulta llamativo que el nuevo proyecto, además de considerar los marcadores tradicionales asociados a la biometría como dato personal, precontemple adicionalmente indicaciones (art. 16 quáter) para el tratamiento de datos personales relativos al perfil biológico humano. Esto implica los legisladores consideran también el uso de muestras biológicas, lo cual, como se ha mencionado, corresponde a aplicaciones más recientes. Sin embargo, esto podría ser un tanto limitado respecto del material biológico que considera, tal como se encuentra planteado actualmente, ya que asocia el perfil biológico a datos genéticos, proteómicos, o metabólicos. Acerca de esto, se regula en específico los fines para los cuales se pueden utilizar (diagnóstico médico, asistencia médica o sanitaria en caso de urgencia, estudios científicos que vayan en beneficio de la salud humana, y en cumplimiento de resoluciones judiciales). Asimismo, prohíbe el tratamiento, cesión y almacenamiento de datos de perfil biológico cuando las muestras han sido obtenidas en ámbitos laborales, educativos, deportivos o sociales, salvo que, por ley, se autorice su uso. Se autoriza también su uso en publicaciones científicas, bajo condición de que los datos publicados sean anónimos. En vista de lo anterior, bioseñales que actualmente se proyectan en la aplicación como identificadores (por ejemplo, EEG, ECG, EDA), no son mencionadas en las categorías de muestra biológica que contempla el proyecto, no encontrándose por tanto al amparo de ninguna regulación en su uso como señales biométricas en Chile, ni en el actual marco legal ni en lo que se ha propuesto en el proyecto en revisión actualmente.

\section{Consideraciones bioéticas internacionales res- pecto de la biometría}

En las últimas dos décadas, diversas comisiones y documentos han revisado las prácticas respecto de la aplicación de biometría, y han puesto de relieve las principales consideraciones éticas para su uso.

Un primer antecedente surge en 2001, con el informe RAND(13), desarrollado por el ejército de Estados Unidos posterior a los sucesos del 11 de septiembre de 2001. Se analizan allí los problemas éticos, legales y sociológicos que surgen con el uso de la biometría. Se especifican tres principales áreas en términos de consideraciones éticas: 1) privacidad de la información; 2) privacidad física; 3 ) objeciones religiosas. Estas tres áreas constituirían riesgos latentes. El riesgo a la privacidad de la información redunda en que la información pueda ser usada de modo más amplio que el que fue originalmente previsto (function creep); que se realice 
un seguimiento y monitoreo permanente de los movimientos físicos de los individuos en tiempo real, y el robo de la identidad. A su vez, el riesgo para la privacidad física se asociaría con el eventual estigma que pudiese surgir a partir de la medida biométrica (en controles fronterizos, por ejemplo, donde algunos individuos pudiesen sentirse amedrentados al solicitárseles la biometría), siendo muchas veces asociada, por ejemplo, la huella dactilar al ámbito criminal forense. También el potencial daño que pudieran sufrir los individuos por la tecnología misma, y la eventual falta de higiene que pudiese existir en algún dispositivo utilizado para capturar la señal. La objeción religiosa se presenta en dicho informe como un tema idiosincrático sociocultural, ya que, en EE.UU., algunos grupos cristianos aluden a la biometría como la marca del diablo, a partir de ciertas interpretaciones de lo referido en apocalipsis(13:16-18). Aun cuando tal vez no refiera a grupos o creencias extendidas de la sociedad (lo referente a la marca del diablo), el reporte concluye que igualmente corresponde a objeciones que debieran tenerse en cuenta seriamente, en vista del énfasis social y legal del respeto por las distintas creencias.

Un segundo antecedente relevante es el informe publicado en 2004 por la $\operatorname{OECD}(14)$, que se enfoca fundamentalmente en la necesidad de realizar esfuerzos para el máximo aseguramiento de la privacidad de los datos de los individuos. Esto se repite como foco central en distintos documentos de revisión(15:18). Algunas recomendaciones prácticas para el cuidado de la privacidad, y de modo muy especial referidas al uso de la biometría para el monitoreo y vigilancia de la población por asuntos de seguridad, es la pixelación y desenfoque de imágenes a través de filtros de distorsión. Lo mismo se recomienda cuando el registro corresponda a la voz, la cual, aun cuando conserve la identificación del contenido, debe ser completamente irreconocible respecto del emisor.

El mayor foco puesto en la privacidad en los documentos de revisión respecto del uso de biometría podría entenderse porque la identidad personal de los individuos es de tremenda importancia, ya que distingue y valida a la persona como ser individual. Por tanto, también es importante reflexionar acerca de cómo las formas de identidad, ciudadanía y pertenencia, y los modos de identificación, se redefinen y reconfiguran cada vez más en privilegio del desarrollo de estrategias para afrontar el riesgo y la seguridad social, y se hacen susceptibles a diversos modos de control a través de la tecnología. Resulta evidente que, probablemente, las transformaciones asociadas a estos procesos se desarrollan en el campo de la biopolítica, es decir, la gestión de la vida mediante la cual la fusión de cuerpo y tecnología, junto con la segmentación de la sociedad, es precisamente lo que se pone en juego(19). En el contexto de lo mencionado, algunos autores (20) han señalado que los debates actuales en biociencia y biomedicina se dan a partir de una serie de ansiedades. Estas se asocian fundamentalmente con, por ejemplo, evitar prácticas que lleven al racismo biológico o al resurgimiento de la eugenesia a partir de los nuevos avances tecnológicos y los modos de enfrentar los riesgos de modo distribuido ("molecular") en las sociedades actuales. De este modo, se empezaría a configurar una nueva forma de biopolítica — que se ha denominado "ethopolitica" - la cual implica el predominio de una racionalidad política (mezcla de ética y política). Esta llevaría a reconfigurar las relaciones y prácticas de los individuos respecto de sí mismos, hacia las autoridades y hacia el Estado, con un tenor normativo. De este modo, se constituye en orientaciones sobre cómo la vida debe ser vivida, en cuanto previene a los individuos respecto de sus futuros riesgos, para que sean capaces de tomar decisiones informadas sobre su salud y la vida en general, configurando una individualidad primordialmente somática, con énfasis en la experiencia corporal del sí mismo. Este modelo ethopolítico se traduce en individuos a los que se les fomenta que tomen parte activa en ser ciudadanos prudentes y responsables, capaces de asegurar su propia salud y bienestar, llevando a definir un nuevo tipo de ciudadano: el ciudadano biológico. A partir de lo anterior, se puede entender entonces el hecho de que la biometría haya tenido un auge vertiginoso en las últimas décadas, siendo un elemento consonante con la práctica de que el cuerpo sea el objeto de medición, clasificación, control y vigilancia.

\section{Conclusión}

En Chile, el marco legal vigente no ha considerado aún a la biometría como un dato personal, lo cual pone de manifiesto que constituye un elemento de auge vertiginoso reciente, ya que la ley 
actual fue promulgada en 1999. Esto lleva a entender que la biometría, recién en las últimas dos décadas, ha empezado a visibilizarse en las vidas cotidianas de las personas en la realidad nacional. Siendo así, resulta alentador que el proyecto de ley, actualmente en estudio para ser promulgado, lo haya incorporado como un dato personal sensible, habiendo integrado además una definición que delimita lo que resultará atribuible al concepto de "biometría". No obstante, se constata que la propuesta actual adolece de precisión en la definición de las medidas biométricas y resulta limitada en cuanto a las bioseñales que se están implementando y que probablemente tengan mayor expansión en el futuro, tales como el ECG, EEG y EDA.

Actualmente, la adopción de la tecnología biométrica está aumentando rápidamente en distintas aplicaciones, pudiendo prestar utilidad, por ejemplo, en la seguridad ciudadana y en la protección contra el crimen. Sin embargo, su aplicación conlleva de manera latente la preocupación respecto de la violación de la privacidad y los derechos de las personas. Entre los factores que se pueden incluir en este sentido se podría mencionar la posibilidad de fraude, robo de identidad, violaciones a la libertad de los individuos e inexactitud de los datos. Estos riesgos de error o violación de derechos podrían crear conflictos entre aquellos a cargo del tratamiento de los datos personales y los titulares de los datos, ya que, por ejemplo, podrían ser acusados de un delito o ser víctimas de discriminación de manera injusta y en base a evidencia biométrica equívoca. Resulta de gran relevancia además regular el almacenamiento de la base biométrica de datos y los límites en cuanto a compartir los datos personales con entidades que se supone no debieran conocerlos. Esto implica desafíos en cuanto a planificar la seguridad a corto y largo plazo de los bancos de datos biométricos, de tal modo de aminorar la probabilidad de seguimiento de dichos datos personales.

Una adecuada planificación de la administración, de los deberes de los encargados del tratamiento de los datos biométricos, de la claridad en la definición de procedimientos a prueba de fallas en la gestión de los bancos de datos, garantizarán que existan aplicaciones basadas en la biometría que cumplan con los estándares y requisitos éticos para el respeto, tanto de los derechos de los individuos como de los derechos colectivos en el futuro, en un mundo donde la biotecnología y la noción de ciudadanos biológicos respete la particularidad y no conduzca a la despersonalización y difusión identitaria en una biomasa. 
Aspectos éticos del uso de identificadores biométricos - Boris A. Lucero M. et al.

\section{Referencias}

1. Jain AK, Flynn P, Ross AA. Handbook on Biometrics, New York, USA: Springer Science \& Business Media; 2008.

2. Fraden J. Handbook of Modern Sensors: Physics, Designs, and Applications, $3^{\text {rd }}$ Edition. NewYork, USA: Springer Science \& Business Media, Inc.; 2004.

3. Ouwerkerk M, Pasveer F, Langereis G. Unobtrusive Sensing of Psychophysiological Parameters: Some Examples of Non-Invasive Sensing Technologies, Vol. 8 of Philips Research Book Series. Dordrecht, The Netherlands: Springer, 2008.

4. Hao Y, Foster R. Wireless body sensor networks for health monitoring applications. Physiological Measurement 2008; 29(11): R27-R56.

5. Mordini E, Petrini C. Ethical and social implications of biometric identification technology. Annali dell'Istituto superiore di sanita 2007; 43(1): 5-11.

6. Jonietz E. Il boom della biometria. Techn. Rev. 2004; 16(6): 12-3.

7. Basar A. Brain Function and Oscillations: Integrative Brain Function. Neurophysiology and Cognitive Processes (Springer series in synergetics). Berlin, Germany: Springer-Verlag; 1999.

8. Klonovs J, Petersen C, Olesen H, Hammershoj A. ID proof on the go: Development of a mobile EEG-based biometric authentication system. IEEE Veh. Technol. Mag. 2013; 8(1), 81-89.

9. Campisi P, La Rocca D. Brain waves for automatic biometric-based user recognition. IEEE transactions on information forensics and security 2014; 9(5), 782-800.

10. De Leeuw K, Bergstra J. The History of Information Security: A Comprehensive Handbook. Amsterdam, The Netherlands: Elsevier B.V.; 2007.

11. República de Chile. Ley 19628 de 28 de Agosto de 1999, de Protección de la vida privada. Biblioteca del Congreso Nacional de Chile/BCN

12. Boletín $\mathrm{N}^{\circ} 11.092 .07$ de 17 de Enero de 2017, Proyecto de ley de Protección de los datos personales. Disponible en https://www.camara.cl/pley/pley_detalle.aspx?prmID=11608\&prmBoletin=11092-07

13. Woodward JD Jr, Webb KW, Newton EM, Bradley M, Rubenson D, et al. Army biometric applications: identifying and addressing sociocultural concerns, Santa Monica (CA): RAND; 2001.

14. OECD. Biometric-based Technologies. OECD Digital Economy Papers 2004; 101. OECD Publishing, Paris. Disponible en http://dx.doi.org/10.1787/232075642747.

15. European Commission Joint Research Center. Biometrics at the frontiers: Assessing the impact on society. Brussels: EC; September 2005. (EC-DG JRC-IPTS, 2005).

16. National Science and Technology Council. The national bio-metrics challenge. Washington DC: NSTC; 2006.

17. National Science and Technology Council. Biometrics foundation documents. Washington DC: NSTC; 2006.

18. National Science and Technology Council. Privacy \& biometrics: building a conceptual foundation. Washington DC: NSTC; 2006.

19. Ajana B. Governing through biometrics: The biopolitics of identity. Springer; 2013.

20. Rose N. The politics of life itself. Theory, culture \& society 2001 Dec;18(6): 1-30.

Recibido: 2 de mayo de 2019

Aceptado: 5 de junio de 2019 